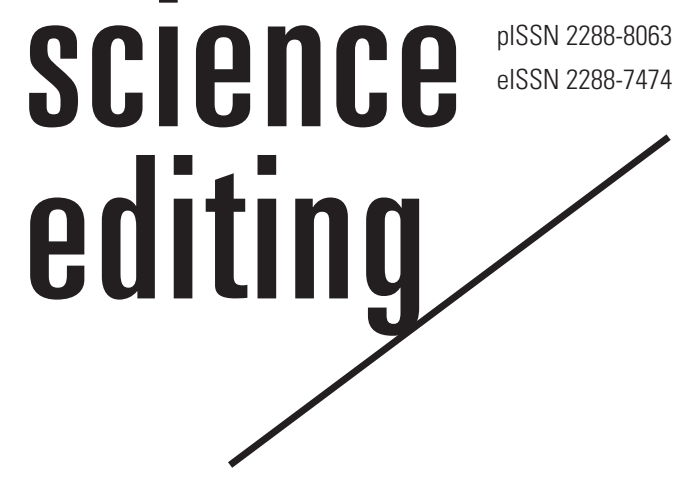

\title{
Bibliometric analysis of publications on inclusive education from the Web of Science Core Collection published from 1992 to 2020
}

\author{
Jia-Fen $\mathrm{Wu}^{1}$, Xiaoxiao Lin $^{2}$ \\ 'Department of Early Childhood Education, School of Education, JingGangShan University, Ji'an; ${ }^{2}$ Department of Special \\ Education, School of Education, Guangzhou University, Guangzhou, China
}

\section{Abstract}

Purpose: This study analyzed the bibliometric characteristics of publications on inclusive education in the Social Science Citation Index and Science Citation Index Expanded in the Web of Science Core Collection from 1992 to 2020.

Methods: Terms related to "inclusive education" and "inclusion of education" were used as keywords to search for journal articles on July 3, 2020.

Results: There were 1,786 articles, representing 3,376 authors, in the 345 journals scanned. The United States, United Kingdom, and Australia were the three leading countries/regions in this field. In the top 12 countries, the top 15 institutions and the top 10 most-cited journals were identified by either the number of publications or the number of total citations. Core themes from the 30 most highly-cited articles were teachers' attitudes, teachers' self-efficacy, and the effects of inclusive education. Teachers included both pre-service and in-service teachers; students represented those with and without special educational needs.

Conclusion: The results indicate that the United States, United Kingdom, and Australia dominated inclusive education research, originating most of the highly-cited articles, having more prolific authors, and presenting the most-cited institutions. Furthermore, three emerging core themes from the 30 most highly-cited articles were teachers' attitudes, teachers' self-efficacy, and the effects of inclusive education. Frontline teachers are recommended to submit manuscripts about their teaching experiences to the most-cited journals, which have a large readership. To measure the effects of inclusive education, it is essential to formulate reliable, valid, and culturefree research instruments for future studies.

Correspondence to Xiaoxiao Lin 104438@gzhu.edu.cn

\section{ORCID}

Jia-Fen Wu

https://orcid.org/0000-0003-1097-221X Xiaoxiao Lin

https://orcid.org/0000-0002-3624-3544
Keywords

Bibliometric analysis; Histcite Pro 2.0; Historiograph; Inclusive education; Total Local Citation 


\section{Introduction}

Background/rationale: Inclusive education was ratified in the UNESCO (United Nations Educational, Scientific and Cultural Organization) Salamanca Statement and Framework for Action in Special Needs Education in 1994. Since inclusive education emphasizes placing students with special needs in age-appropriate general education classes or mainstream schools and enables them to succeed [1,2], it is seen as "an issue of social justice as well as an issue of equity" [3] and as one of the most effective means of combatting educational inequity.

With the advent of inclusive education practices, advocates for inclusive education believe that all students, with and without learning disabilities, may potentially receive highquality instruction and interventions; however, the long-term effects of inclusive education remain controversial, despite the increasing number of publications on this topic.

Purpose: We sought to map inclusive education knowledge through a holistic bibliometric evaluation, including annual publications, most-cited countries, most-cited journals, mostcited institutions, core themes, and potential trends.

\section{Methods}

Ethics statement: Neither institutional review board approval nor informed consent was required because this study was based on a literature database.

Study design: This study conducted a bibliometric analysis of a specific topic from a literature database.

Data/measurement: Data related to the present study were retrieved from the core collection of Web of Science on July 3, 2020. Two terms ("inclusive education" and "inclusion of edu- cation") were used as the keywords for searching journal articles. Only publications published from 1992 to 2020 were considered. The timespan was set as "ALL YEAR" to thoroughly retrieve related data from the past. There are 1,786 articles (including early-access articles), representing 3,376 authors, in the 345 journals that were scanned. A total of 54,747 references with 2,981 keywords were included in the titles and abstracts (Dataset 1).

Visualization: Data obtained from Web of Science were exported to Histcite Pro 2.0, which was used to visualize bibliometric maps related to scientific affairs [4] and to profile the citation relationships among articles in the historiograph.

Total Local Citation Score: The Total Local Citation Score (TLCS) referred to the total number of citations from scholars in the field of inclusive education within the collected datasets.

\section{Results}

Annual number of articles: As shown in Fig. 1, the first article appeared in 1992 and then the growth rate showed a zigzag pattern of instability from 1995 to 2005. Since 2006, the number of publications has gradually increased. Since 2012, the annual publication volume has exceeded 100 , and the highest number of publications was found in 2019.

Countries of publications: A total of 99 countries were counted, and only the top 12 countries were listed. The top 12 countries are presented in Fig. 2. The United Kingdom, which ranked first based on the TLCS, contributed the most original articles $(292,16.3 \%$ of the 1,786$)$, followed by the United States (379, 21.2\%), Australia (235, 13.2\%), South Africa (134, $7.5 \%)$, Canada (89,5\%), and the People’s Republic of China $(89,5 \%)$, respectively. The United States had the most publi-

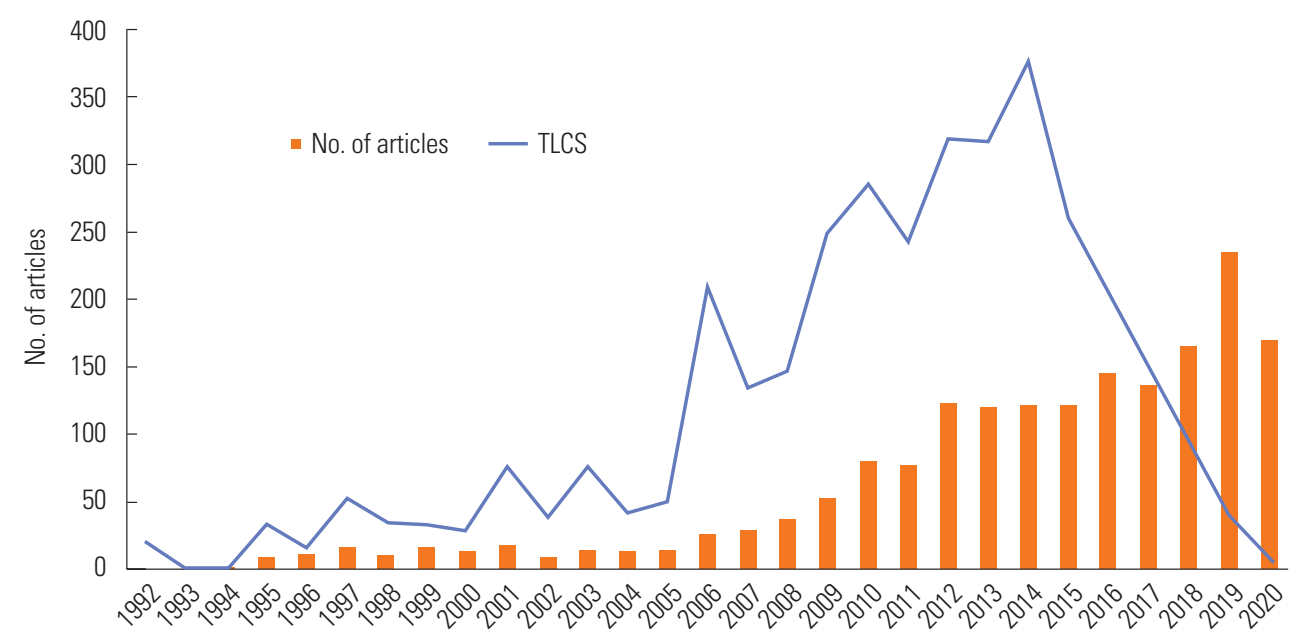

Fig. 1. Annual distribution of publications on inclusive education. TLCS, Total Local Citation Score. 
cations, followed by the United Kingdom and then Australia; these three leading countries accounted for $50.7 \%$ of the 1,786 articles. Canada and China had the same amount of publications, but Canada had more citations. Six of the top countries are in Europe. China and Cyprus are in Asia, the United States of America and Canada are in America, Australia is in Oceania, and South Africa is in Africa.

Institutions of authors: As shown in Fig. 3, Monash University produced the most articles (46 articles), followed by North West University (38), Queensland University of Technology (37), the University of Jyvaskyla (35), and the University of Kansas (31). Monash University ranked first in citations (TLCS, 174), followed by North West University (TLCS, 158) and the University of Manchester (TLCS, 158), Hong Kong Institute of Education (TLCS, 156), and the University of Eastern Finland (TLCS, 129). Most-cited journals: The 10 most-cited journals with at least 300 total citations are listed in Fig. 4. The top five were the International Journal of Inclusive Education (465 articles), European Journal of Special Needs Education (97 articles), International

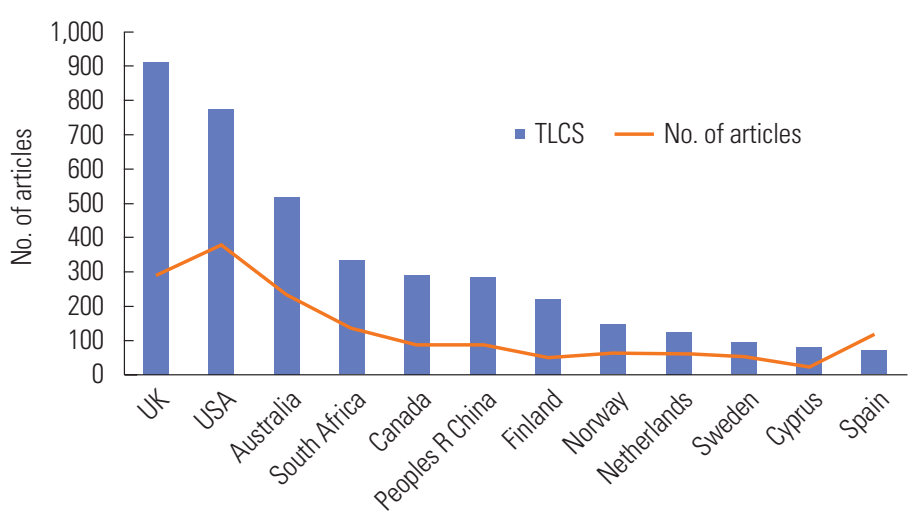

Fig. 2. Top 12 countries. TLCS, Total Local Citation Score.
Journal of Disability Development and Education (75 articles), Disability \& Society (61 articles), and Teaching and Teacher Education (56 articles). According to TLCS, the International Journal of Inclusive Education had the most citations $(1,010)$, followed by European Journal of Special Needs Education (270), Teaching and Teacher Education (226), Disability \& Society (154), and Research and Practice for Persons with Severe Disabilities (117). The European Journal of Psychology of Education, with only nine articles, ranked eighth and Exceptional Children, with only eight articles, ranked 10th.

Most-cited authors: Fig. 5 shows the top 12 most-cited authors by articles and citations. Sharma, U. published the most articles, but ranked seventh in citations. Engelbrecht, P. ranked first in citations, but ranked fourth in the number of articles. Despite only publishing only six articles, Singal, N. ranked 10th in citations.

Citation relationship of articles: The historiograph shown in Fig. 6 presents the citation relationship of the top 30 most-cited articles extracted by their numerical citations in 345 journals and in the direct citations made between those 1786 articles. Of the 30 most-cited articles, the most significant articles were written by Savolainen et al. (466) [5], Forlin et al. (252) [6], Ruijs et al. (245) [7], and Parasuram (156) [8].

Core themes: The following core themes emerged teachers' attitudes towards inclusive education, pre-service and in-service teachers' self-efficacy, the effects of inclusive education, and educational policies.

\section{Discussion}

Interpretation: A total of 1,786 articles on inclusive education were published during the past 29 years, from 1992 to 2020. The amount of publications gradually increased after 2006

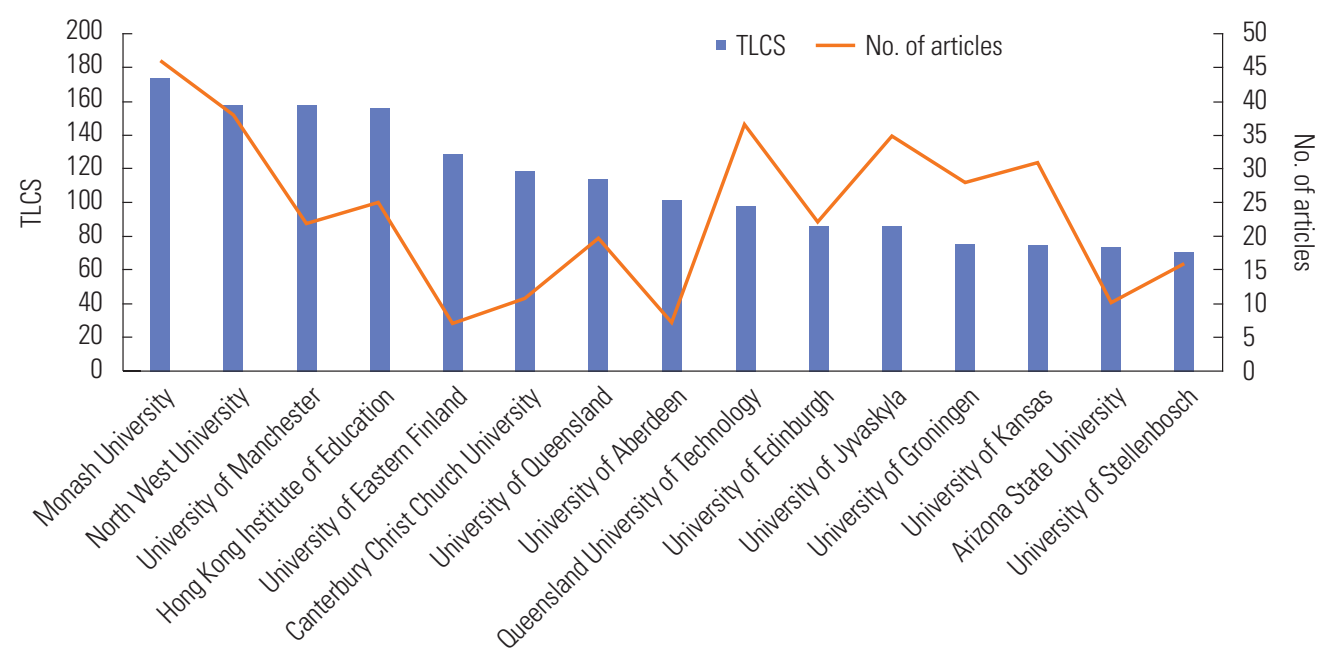

Fig. 3. Top 15 most-cited institutions by articles and citations. TLCS, Total Local Citation Score. 


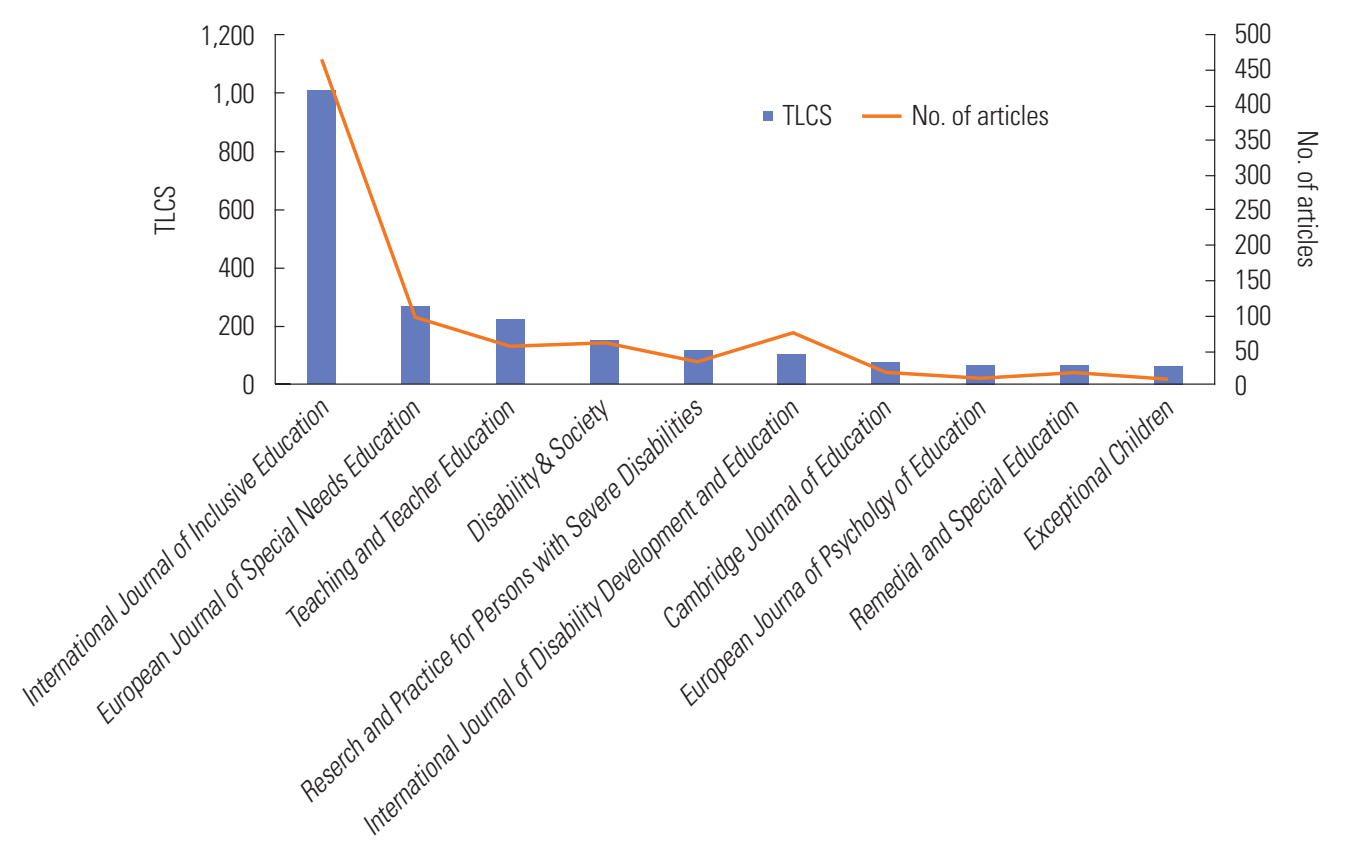

Fig. 4. Top 10 most-cited journals. TLCS, Total Local Citation Score.

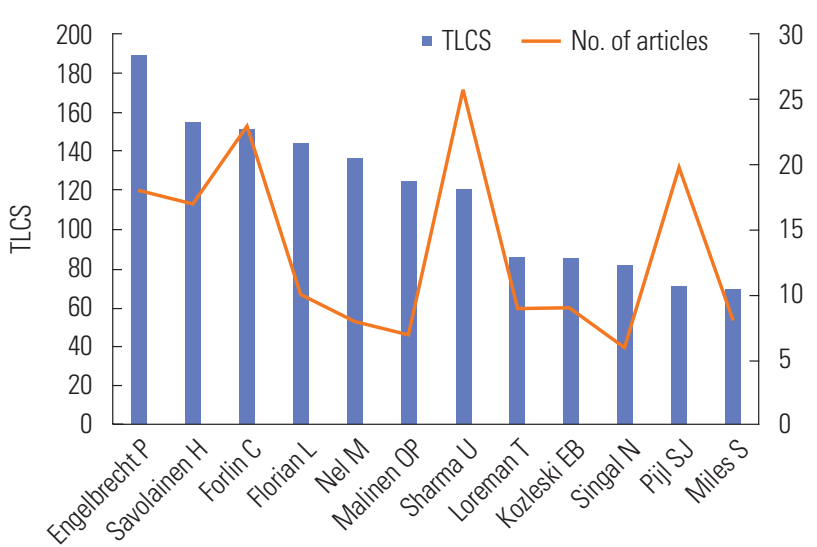

Fig. 5. Top 12 most-cited authors by articles and citations. TLCS, Total Local Citation Score.

and then dramatically increased from 2012 onwards. The publications between 2012 and 2020 accounted for $74.8 \%$ of the total of 1786 articles (Fig. 1). The most productive publication interval was from 2018 to 2019. The United States was found to be the country with the most publications, while the United Kingdom was the leading country in terms of citations (Fig. 2).

Out of the top 15 institutions, four institutions are located in the United Kingdom, three in Australia, two in South Africa, two in the United States, two in Finland, one in the Netherlands, and one in China (Fig. 3). Most institutions are located in the leading countries. Seven of the top 15 institutions

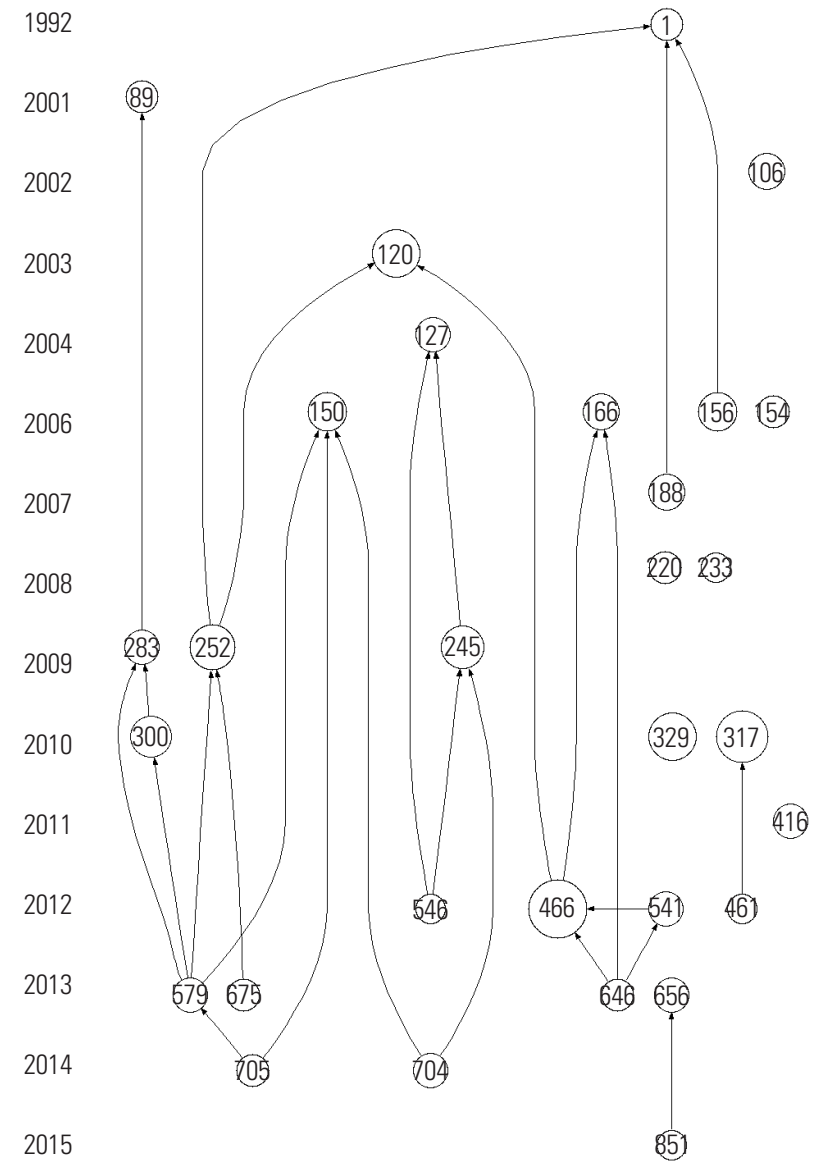

Fig. 6. Historiograph of the top 30 articles. 
were found to be in Europe, three in Oceania, two in the America, two in Africa, and one in Asia. These top 10 mostcited journals in Fig. 4 are attributed to two clusters: cluster 1 includes special education-related journals, such as the International Journal of Inclusive Education, European Journal of Special Needs Education, and Disability \& Society; cluster 2 includes education-related journals, such as Teaching and Teacher Education, Cambridge Journal of Education, and European Journal of Psychology of Education.

One leading article, "Variables that affect teachers' attitudes towards disability and inclusive education in Mumbai, India" [8], explored the variables that influenced teachers' attitudes towards children's disability and inclusive education. To a certain extent, teachers' attitudes are vital for the effectiveness of implementing inclusive education. The most-cited articles in 2009 focused on demographic variables in terms of changing pre-service teachers' attitudes and sentiments [6]. In addition, scholars were interested in the real effects of inclusive education on students with and without special educational needs [7]. Both teachers' attitudes and self-efficacy in inclusive education are important and should be included in pre-service and in-service teacher education [5].

Limitations: First, only bibliographic data from the Web of Science were selected; thus, some relevant publications might have been overlooked in this study. Second, the TLCS values were inherently affected by the time elapsed since article publication. Third, the historiograph generated by Histcite Pro 2.0 cannot display international collaboration, and few internationally collaborative publications were found in this study. Last, since the literature search was conducted in July 2020, the data for 2020 lacked completeness and were not representative. Based on the above limitations, further research might expand the scope of databases to include others, such as Scopus or ERIC, and average citations per year is recommended as an indicator for future research. Visualization software such as VOSviewer and CiteSpace can be applied to map collaborative networks among countries, institutions, and authors; furthermore, data for the full year of 2020 can be retrieved in the future to update the completeness of this study.

Core themes from the most highly-cited articles included teachers' attitudes, teachers' self-efficacy, and the effects of inclusive education. Teachers included both pre-service and inservice teachers; students represented those with and without special educational needs.

Conclusion: The results of this study indicate that the United Kingdom, the United States, and Australia dominated inclusive education research, originating most of the highly-cited articles, having more prolific authors, and presenting the most-cited institutions. Furthermore, three core themes emerged from the 30 most highly-cited articles: teachers' attitudes, teachers' self-efficacy, and the effects of inclusive education. Teachers included both pre-service and in-service teachers; students represented those with and without special educational needs. Teachers' attitudes and self-efficacy towards inclusion of education will have significant impacts on the success of practicing inclusive education. Therefore, pre-service teachers need to take special education courses to enhance their pedagogical knowledge, whereas in-service teachers need continuing training to empower their pedagogical ability. The International Journal of Inclusive Education was the most-cited journal in this field and published the most professional articles regarding inclusive education. The most-cited journals also included education-related journals, which accept manuscripts regarding teaching and teacher education. Frontline teachers in general classrooms should consider submitting manuscripts about their teaching experiences to these most-cited journals, which have a large readership. To measure the effects of inclusive education and teachers' self-efficacy, it is necessary to formulate instruments for quantitative or qualitative research, such as questionnaires, scales, and interview protocols. Moreover, the reliability and validity of those research instruments should be carefully calculated. Few collaborative studies of inclusive education were found, but it is possible to conduct cross-cultural studies in the near future. Thus, developing a culture-free research instrument will also be essential.

\section{Conflict of Interest}

No potential conflict of interest relevant to this article was reported.

\section{Funding}

The work was supported by the Foundation of Ministry of Education of Guang Dong Province, China (grant no. GD 17XJY17).

\section{Data Availability}

Data are available from the author upon reasonable request.

Dataset 1. Inclusive education: 1992 to 2020

\section{References}

1. Bui X, Quirk C, Almazan S, Valenti M. Inclusive education, research and practice: inclusion works [Internet]. Elkridge, MD: Maryland Coalition for Inclusive Education; 2010 [cited 2018 Mar 7]. Available from: http://www. 
mcie.org/site/usermedia/application/11/inclusion-works(2010).pdf

2. Alquraini T, Gut D. Critical components of successful inclusion of students with severe disabilities: literature review. Int J Spec Educ 2012;27:42-59.

3. Gerrard LC. Inclusive education: an issue of social justice. Equity Excell Educ 1994;27:58-67. https://doi.org/10.1080/ 1066568940270111

4. De Robbio A, Raman Nair R. Eugene Garfield 1925-2017: informatician nonpareil. Trivandrum: Centre for Informatics Research and Development, Centre for South Indian Studies; 2017.

5. Savolainen H, Engelbrecht P, Nel M, Malinen OP. Understanding teachers' attitudes and self-efficacy in inclusive education: implications for pre-service and in-service teacher education. Eur J Spec Needs Educ 2012;27:51-68. https:// doi.org/10.1080/08856257.2011.613603

6. Forlin C, Loreman T, Sharma U, Earle C. Demographic differences in changing pre-service teachers' attitudes, sentiments, and concerns about inclusive education. Int J Incl Educ 2009;13:195-209. https://doi.org/10.1080/13603110701365356 7. Ruijs NM, Peetsma TT. Effects of inclusion on students with and without special educational needs reviewed. Educ Res Rev 2009;4:67-79. https://doi.org/10.1016/j.edurev.2009.02.002

8. Parasuram K. Variables that affect teachers' attitudes towards disability and inclusive education in Mumbai, India. Disabil Soc 2006;21:231-42. https://doi.org/10.1080/09687590600617352 